\title{
On the metastable homotopy of mod 2 Moore spaces
}

\author{
ROMAN MIKHAILOV \\ JIE WU
}

\begin{abstract}
We study the exponents of metastable homotopy of mod 2 Moore spaces. We prove that the double loop space of $4 n$-dimensional mod 2 Moore spaces has a multiplicative exponent 4 below the range of 4 times the connectivity. As a consequence, the homotopy groups of $4 n$-dimensional mod 2 Moore spaces have an exponent of 4 below the range of 4 times the connectivity.
\end{abstract}

55Q52, 14F35, 55Q20; 55Q05, 55P35

\section{Introduction}

Metastable homotopy has been studied by various people since the early 1950s; see M G Barratt [1], Barratt and Mahowald [3], Baues [4], Green and Holzsager [12], James [13], Mahowald [15; 16], Morisugi [17] and Tipple [19]. The descriptions on the lower metastable homotopy groups of the Moore spaces given by Barratt [1] in 1954 led to computational results announced in [19]. In this article, we consider the exponents of the metastable homotopy groups of mod 2 Moore spaces.

Let $P^{n}(2)=\Sigma^{n-2} \mathbb{R} \mathrm{P}^{2}$ be the $n$-dimensional $\bmod 2$ Moore space with $n \geq 3$. It is well known that $P^{n}(2)$ has a suspension exponent 4 , that is, the degree-4 map [4]: $P^{n}(2) \rightarrow P^{n}(2)$ is nullhomotopic. By the classical result of Barratt [2], the metastable homotopy of $P^{n}(2)$ has an exponent dividing 8 . This leads to the natural question of whether the metastable homotopy of $P^{n}(2)$ has an exponent 4 . The answer to this question is negative for the cases $n \equiv 2,3 \bmod 4$ because there are $\mathbb{Z} / 8$-summands occurring in the lower metastable homotopy groups, according to Cohen and $\mathrm{Wu}$ [10], Morisugi and Mukai [18] and Tipple [19]. The purpose of this article is to give an affirmative answer to this question for the case $n \equiv 0 \bmod 4$ with $n>5$.

We actually show that the double loop space $\Omega^{2} P^{n}(2)$ has the multiplicative exponent 4 below the range roughly four times the connectivity in the case $n \equiv 0 \bmod 4$ with $n>5$. More explicitly, our main result is the following. 
Theorem 1.1 Let $n \equiv 0 \bmod 4$ with $n>4$. Then the power map

$$
\text { 4: } \Omega^{2} P^{n}(2) \rightarrow \Omega^{2} P^{n}(2)
$$

restricted to the skeleton $\operatorname{sk}_{4 n-9}\left(\Omega^{2} P^{n}(2)\right)$ is nullhomotopic.

As a consequence, we get the description of exponents of the homotopy groups up to the given range; for the first case, $n=4$, the homotopy groups $\pi_{k}\left(P^{4}(2)\right)$ are known up to the range $4 n-4[21]$.

Corollary 1.2 Let $n \equiv 0 \bmod 4$. Then $4 \cdot \pi_{k}\left(P^{n}(2)\right)=0$ for $k<4 n-7$.

It is unknown whether the homotopy groups of mod 2 Moore spaces have a bounded exponent. It is known that there are infinitely many $\mathbb{Z} / 8$-summands (in different dimensions) occurring in the homotopy groups of mod 2 Moore spaces; see Cohen and $\mathrm{Wu}[10]$. Our result shows that the first $\mathbb{Z} / 8$-torsion should occur in the range at least four times the connectivity in the case $n \equiv 0 \bmod 4$.

We briefly sketch the proof of Theorem 1.1 as follows. We use the Cohen groups for displaying the explicit obstructions to the $4^{\text {th }}$ power map on the single loop space $\Omega P^{n}(2)$. By using the shuffle relations and the Hopf invariants on general configuration spaces, the $4^{\text {th }}$ power on the double loop space $\Omega^{2} P^{n}(2)$ up to the given range is decomposed as a composite involving the Whitehead product. After handling the reduced evaluation map, Theorem 1.1 is then proved via Theorem 3.6 in the special case when the Whitehead square $\omega_{n-1}$ is divisible by 2 . With the help of technical lemmas about Whitehead products on $P^{4 n}(2)$, Theorem 1.1 is finally proved using Theorem 5.3. The key lemma (Lemma 4.3) presents a special property for $4 n-$ dimensional mod 2 Moore spaces, which is hinted at by Mark Mahowald's result [14] that $\left[\iota_{4 n-1}, \eta_{4 n-1}\right]=0$.

The article is organised as follows. In Section 2, we discuss the $4^{\text {th }}$ power map on the single loop spaces and double loop spaces. The reduced evaluation map on mod 2 Moore spaces is studied in Section 3, where Theorem 3.6 is the special case of Theorem 1.1 in the case when the Whitehead square is divisible by 2 . We give some lemmas in Section 4. Theorem 1.1 is proved in Section 5, where Theorem 1.1 is restated as Theorem 5.3.

\section{The $4^{\text {th }}$ power map on looped suspensions}

In this section, we display the obstructions to the $4^{\text {th }}$ power map on $\Omega \Sigma^{2} X$ and $\Omega^{2} \Sigma^{2} X$ below four times the connectivity for spaces $\Sigma^{2} X$ having suspension exponent 4 . 


\section{The obstructions to the $4^{\text {th }}$ power map on $\Omega \Sigma^{2} X$}

We use the Cohen groups [8] for computing the obstructions to the $4^{\text {th }}$ power map. Recall that the Cohen group $K_{n}^{\mathbb{Z} / 4}=K_{n}^{\mathbb{Z} / 4}\left(x_{1}, \ldots, x_{n}\right)$ is combinatorially defined by generators $x_{1}, \ldots, x_{n}$ with the following relations:

(1) iterated commutators are given by $\left[\left[x_{i_{1}}, x_{i_{2}}\right], \ldots, x_{i_{t}}\right]=1$ if $x_{i_{p}}=x_{i_{q}}$ for some $1 \leq p<q \leq n$, where commutators are defined as $[a, b]=a^{-1} b^{-1} a b$, and

(2) $x_{i}^{4}=1$ for $1 \leq i \leq n$.

Let $d_{i}: K_{n}^{\mathbb{Z} / 4} \rightarrow K_{n-1}^{\mathbb{Z} / 4}$ be the group homomorphism defined by

$$
d_{i}\left(x_{j}\right)= \begin{cases}x_{j} & \text { if } j<i \\ 1 & \text { if } j=i \\ x_{j-1} & \text { if } j>i\end{cases}
$$

The Cohen group $H_{n}^{\mathbb{Z} / 4}$ is defined as the equaliser of the maps $d_{i}: K_{n}^{\mathbb{Z} / 4} \rightarrow K_{n-1}^{Z / 4}$ for $1 \leq i \leq n$. Namely, $H_{n}^{\mathbb{Z} / 4}$ is the subgroup of $K_{n}^{\mathbb{Z} / 4}$ consisting of the words $w$ with the property that $d_{i}(w)=d_{1}(w)$ for $1 \leq i \leq n$. For the spaces $\Sigma^{2} X$ satisfying the hypothesis that

the identity map id $\Sigma_{\Sigma^{2} X}$ is of order 4 in $\left[\Sigma^{2} X, \Sigma^{2} X\right]$,

there is a commutative diagram of groups ${ }^{1}$

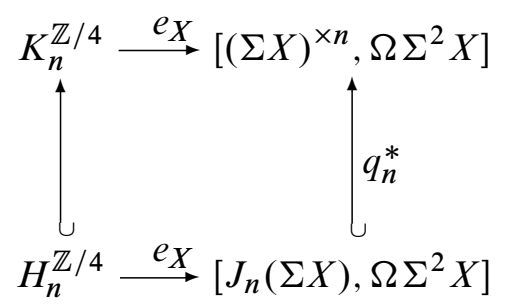

where $J(Y)$ is the James construction with the James filtration $J_{n}(Y)$. The monomorphism in the right column is induced by the quotient map $q_{n}:(\Sigma X)^{\times n} \rightarrow J_{n}(\Sigma X)$ and the group homomorphism $e_{X}$ sends the letter $x_{i}$ to the homotopy class of the composite

$$
(\Sigma X)^{\times n} \stackrel{\pi_{i}}{\longrightarrow} \Sigma X \longleftrightarrow \Omega \Sigma^{2} X,
$$

where $\pi_{i}$ is the $i^{\text {th }}$ coordinate projection for $1 \leq i \leq n$. Let

$$
\alpha_{n}=x_{1} x_{2} \cdots x_{n} \in H_{n}^{\mathbb{Z} / 4} \leq K_{n}^{\mathbb{Z} / 4} .
$$

\footnotetext{
${ }^{1}$ Diagram (2-2) commutes regardless of hypothesis (2-1) for the groups $K_{n}$ and $H_{n}$ integrally without assuming the second relations $x_{i}^{4}=1$ for $1 \leq i \leq n$.
} 
Then $e_{X}\left(\alpha_{n}\right)$ is the homotopy class of the inclusion map $J_{n}(\Sigma X) \rightarrow \Omega \Sigma^{2} X$.

We are only interested in the range below four times the connectivity. It is sufficient to consider only $\alpha_{3}^{4}$, which can be done by direct computations through the Magnus-type representation of $K_{n}^{\mathbb{Z} / 4}$ into the noncommutative exterior algebra $A_{n}^{\mathbb{Z} / 4}$. Here, $A_{n}^{\mathbb{Z} / 4}=A_{n}^{\mathbb{Z} / 4}\left(y_{1}, \ldots, y_{n}\right)$ is the quotient of the tensor algebra $T\left(y_{1}, \ldots, y_{n}\right)$ over $\mathbb{Z} / 4$ by the relations

$$
y_{i_{1}} y_{i_{2}} \cdots y_{i_{t}}=0 \quad \text { if } y_{i_{p}}=y_{i_{q}} \text { for some } 1 \leq p<q \leq t .
$$

The representation $e: K_{n}^{\mathbb{Z} / 4} \rightarrow A_{n}^{\mathbb{Z} / 4}$ is given by $e\left(x_{i}\right)=1+y_{i}$. It is proved in [8] that this is a faithful representation of $K_{n}^{\mathbb{Z} / 4}$.

Now $e\left(\alpha_{3}^{4}\right)=\left(\left(1+y_{1}\right)\left(1+y_{2}\right)\left(1+y_{3}\right)\right)^{4}$ in $A_{3}^{\mathbb{Z} / 4}$. Note that

$$
\left(1+y_{1}\right)\left(1+y_{2}\right)\left(1+y_{3}\right)=1+\sigma_{1}+\sigma_{2}+\sigma_{3},
$$

where

$$
\sigma_{1}=y_{1}+y_{2}+y_{3}, \quad \sigma_{2}=y_{1} y_{2}+y_{1} y_{3}+y_{2} y_{3}, \quad \sigma_{3}=y_{1} y_{2} y_{3} .
$$

Let $\Delta=\sigma_{1}+\sigma_{2}+\sigma_{3}$. Then

$$
e\left(\alpha_{3}^{4}\right)=(1+\Delta)^{4}=1+4 \Delta+6 \Delta^{2}+4 \Delta^{3}+\Delta^{4}=1+2 \Delta^{2}
$$

in $A_{3}^{\mathbb{Z} / 4}$, since $4 \alpha=0$ for $\alpha \in A_{n}^{\mathbb{Z} / 4}$ and $\Delta^{4} \in I^{4} A_{3}^{\mathbb{Z} / 4}=0$, the 4-fold product of the augmentation ideal $I A_{3}^{\mathbb{Z} / 4}$. By using the property that $I^{4} A_{3}^{\mathbb{Z} / 4}=0$, we have $\Delta^{2}=\sigma_{1}^{2}+\sigma_{2} \sigma_{1}+\sigma_{1} \sigma_{2}$. Using the notation $[\alpha, \beta]=\alpha \beta-\beta \alpha$ for $\alpha, \beta$ in an algebra $A$ and using the relations (2-3), we have

$$
\begin{aligned}
2 \sigma_{1}^{2} & =2\left(y_{1}+y_{2}+y_{3}\right)^{2} \\
& =2\left(y_{2} y_{1}+y_{3} y_{1}+y_{1} y_{2}+y_{3} y_{2}+y_{1} y_{3}+y_{2} y_{3}\right) \\
& =2\left(\left[y_{1}, y_{2}\right]+\left[y_{1}, y_{3}\right]+\left[y_{2}, y_{3}\right]\right), \\
2\left(\sigma_{1} \sigma_{2}+\sigma_{2} \sigma_{1}\right) & =2\left(2 y_{1} y_{2} y_{3}+y_{2} y_{3} y_{1}+y_{1} y_{3} y_{2}+y_{2} y_{1} y_{3}+y_{3} y_{1} y_{2}\right) \\
& =2\left(y_{2} y_{3} y_{1}+y_{1} y_{3} y_{2}+y_{2} y_{1} y_{3}+y_{3} y_{1} y_{2}\right) \\
& =2\left(y_{2}\left(\left[y_{1}, y_{3}\right]\right)+\left[y_{1}, y_{3}\right] y_{2}\right) \\
& =2\left[\left[y_{1}, y_{3}\right], y_{2}\right] .
\end{aligned}
$$

(Note that, since we are working modulo 4 , the sign \pm on the terms can be ignored after multiplying by 2.) Using the property [22, Lemma 1.4.8] that $e\left(\left[\left[x_{i_{1}}, x_{i_{2}}\right], \ldots, x_{i_{t}}\right]\right)=$ $1+\left[\left[y_{i_{1}}, y_{i_{2}}\right], \ldots, y_{i_{t}}\right]$, we get

$$
\alpha_{3}^{4}=\left(\left[x_{1}, x_{2}\right]^{2}\left[x_{1}, x_{3}\right]^{2}\left[x_{2}, x_{3}\right]^{2}\right) \cdot\left[\left[x_{1}, x_{3}\right], x_{2}\right]^{2} \text {. }
$$


This method is valid for computing $\alpha_{n}^{4}$ for small $n$. A more effective method for determining $\alpha_{n}^{4}$ for general $n$ can be seen in [9]. The geometric interpretation of formula (2-4) through the representation $e_{X}$ gives the following lemma.

Lemma 2.1 (obstruction lemma) Let $X$ be a $C W$-complex such that $4 \cdot\left[\operatorname{id}_{\Sigma^{2} X}\right]=0$ in $\left[\Sigma^{2} X, \Sigma^{2} X\right]$. Let $\left.4\right|_{J_{3}}: J_{3}(\Sigma X) \rightarrow \Omega \Sigma^{2} X$ be the restriction of the power map 4: $J(\Sigma X) \simeq \Omega \Sigma^{2} X \rightarrow \Omega \Sigma^{2} X$. Then there is a decomposition

$$
\left[\left.4\right|_{J_{3}}\right]=\zeta_{2} \cdot \zeta_{3}
$$

in the group $\left[J_{3}(\Sigma X), \Omega \Sigma^{2} X\right]$, where $\zeta_{2}$ is represented by the composite

$$
J_{3}(\Sigma X) \hookrightarrow J(\Sigma X) \stackrel{H_{2}}{\longrightarrow} J\left((\Sigma X)^{\wedge 2}\right) \stackrel{\Omega W_{2}^{2}}{\longrightarrow} \Omega \Sigma^{2} X,
$$

with $H_{k}$ the $k^{\text {th }}$ James-Hopf invariant and $W_{k}$ the $k$-fold Whitehead product, and $\zeta_{3}$ is represented by the composite

$$
J_{3}(\Sigma X) \stackrel{\text { pinch }}{\longrightarrow}(\Sigma X)^{\wedge 3} \stackrel{\tau_{2,3}}{\longrightarrow}(\Sigma X)^{\wedge 3} \stackrel{S_{3}^{2}}{\longrightarrow} \Omega \Sigma^{2} X,
$$

with $\tau_{2,3}$ the map switching positions 2 and 3 in the self-smash product and $S_{k}$ the $k$-fold Samelson product.

\section{The elimination of the obstruction $\zeta_{3}$}

Consider the looping homomorphism

$$
\Omega:\left[J_{3}(\Sigma X), \Omega \Sigma^{2} X\right] \rightarrow\left[\Omega J_{3}(\Sigma X), \Omega^{2} \Sigma^{2} X\right], \quad[f] \mapsto[\Omega f] .
$$

The obstruction $\zeta_{3}$ can be eliminated after looping using the shuffle relations introduced in [22]. Here we give a proof by highlighting the ideas of the shuffle relations.

Proposition 2.2 The element $\zeta_{3}$ lies in the kernel of the looping homomorphism defined as above. Thus, for any space $Z$ and any map $f: \Sigma Z \rightarrow J_{3}(\Sigma X)$, the composite $\zeta_{3} \circ f: \Sigma Z \rightarrow \Omega \Sigma^{2} X$ is nullhomotopic.

Proof Let $Y=\Sigma X$. Let $J(Y) \wedge J(Y)$ be filtered by

$$
\operatorname{Fil}_{n}(J(Y) \wedge J(Y))=\bigcup_{i+j \leq n} J_{i}(Y) \wedge J_{j}(Y) .
$$

Since $Y$ is a co- $H$-space, there exists a filtration-preserving map

$$
\bar{\psi}: J(Y) \rightarrow J(Y) \wedge J(Y)
$$


such that $\bar{\psi}$ is homotopic to the reduced diagonal $\bar{\Delta}$. Consider the homotopy commutative diagram

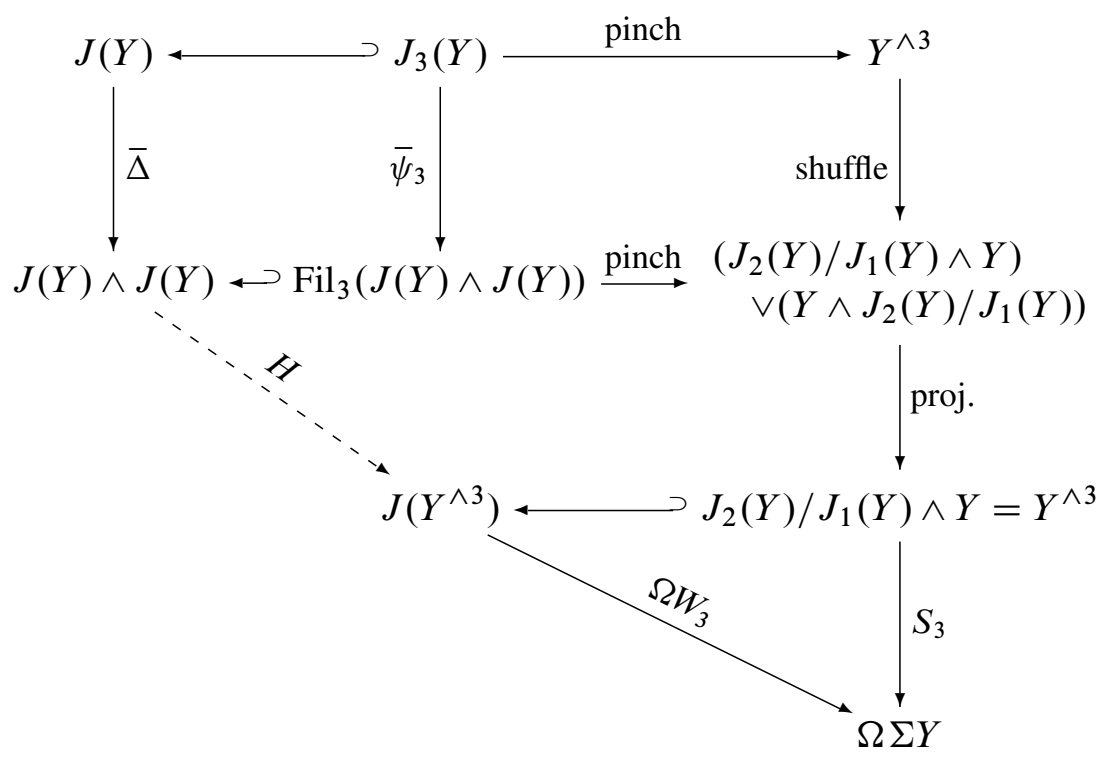

where $S_{3}$ is the Samelson product and the extension map $H$ exists by the suspension splitting of $J(Y) \wedge J(Y)$. Using the Cohen program, we see that the composite from $J_{3}(Y)$ going through the right column represents the element

$$
\left[\left[x_{1}, x_{2}\right], x_{3}\right]+\left[\left[x_{2}, x_{1}\right], x_{3}\right]+\left[\left[x_{3}, x_{1}\right], x_{2}\right]=-\left[\left[x_{1}, x_{3}\right], x_{2}\right]
$$

in the Cohen group $K_{3}$. Note that $-\left[\left[x_{1}, x_{3}\right], x_{2}\right]$ represents a map $S_{3} \circ \tau_{2,3}$ which is $-\frac{1}{2}$ of the map $\zeta_{3}$ in Lemma 2.1. The assertion follows by letting the composite from $J_{3}(Y)$ take the path from the right-hand side and using the property that $\Omega \bar{\Delta}: \Omega J(Y) \rightarrow \Omega(J(Y) \wedge J(Y))$ is nullhomotopic.

\section{The configuration spaces and the obstruction $\zeta_{2}$}

The obstruction $\zeta_{2}$ is essential after looping in general. We use configuration spaces for reducing the obstruction $\zeta_{2}$ from the cubic range to the quadratic range. We refer to C-F Bödigheimer's work [5] as a reference on configuration space models for mapping spaces as well as his constructions of the Hopf invariants on configuration spaces.

Let $M$ be a smooth manifold, $M_{0}$ a submanifold, and $X$ a pointed CW-complex. Let $C\left(M, M_{0} ; X\right)$ be the configuration space with labels in $X$ in the sense of [5] with the filtration $C_{n}=C_{n}\left(M, M_{0} ; X\right)$ induced by the configuration length. Let $D_{n}=D_{n}\left(M, M_{0} ; X\right)$ denote $C_{n} / C_{n-1}$. We will use the following properties: 
(1) [5, Lemma, page 178] Let $N$ be a codimension-zero submanifold of $M$. Then the isotopy cofibration

$$
\left(N, N \cap M_{0}\right) \longrightarrow\left(M, M_{0}\right) \longrightarrow\left(M, N \cup M_{0}\right)
$$

induces a quasifibration

$$
C\left(N, N \cap M_{0} ; X\right) \longrightarrow C\left(M, M_{0} ; X\right) \longrightarrow C\left(M, N \cup M_{0} ; X\right)
$$

provided that $\left(N, N \cap N_{0}\right)$ or $X$ is connected.

(2) [5, Section 3] Let $V=\bigvee_{k=1}^{\infty} D_{k}$. There is a Cohen construction [6] of a power set map $P: C\left(M, M_{0} ; X\right) \rightarrow C\left(\mathbb{R}^{\infty} ; V\right)$, which is natural on $\left(M, M_{0}\right)$ and $X$, inducing a stable splitting of $C\left(M, M_{0} ; X\right)$. The Hopf invariant is given by the composite

$$
H_{k}: C\left(M, M_{0} ; X\right) \stackrel{P}{\longrightarrow} C\left(\mathbb{R}^{\infty} ; V\right) \stackrel{\text { proj. }}{\longrightarrow} C\left(\mathbb{R}^{\infty} ; D_{k}\right)
$$

for $k \geq 1$.

In particular, if $I=[0,1]$, there is a quasifibration

$C([0,1] \times I ; X) \longrightarrow C(([0,3],[2,3]) \times I ; X) \longrightarrow C(([0,3],[0,1] \cup[2,3]) \times I ; X)$ for any path-connected CW-complex $X$ with

$$
C(([0,3],[2,3]) \times I ; X) \simeq * \text { and } C([0,1] \times I ; X) \simeq \Omega^{2} \Sigma^{2} X .
$$

We define the evaluation map

$$
\Sigma \Omega^{2} \Sigma^{2} X \simeq \Sigma C\left(I^{2} ; X\right) \rightarrow \Omega \Sigma^{2} X \simeq J(\Sigma X)
$$

by composing

$$
\Sigma C\left(I^{2} ; X\right) \simeq C\left(\left([0,3], \partial_{+}\right) \times I ; X\right) / C(I \times I ; X) \stackrel{\text { pinch }}{\longrightarrow} C(([0,3], \partial) \times I ; X)
$$

(where $\partial_{+}=[2,3]$ and $\partial=[0,1] \cup[2,3]$ ) with the homotopy inverse of the composite

$$
J(\Sigma X) \stackrel{\simeq}{\longrightarrow} C(I ; \Sigma X) \stackrel{\simeq}{\longrightarrow} C(([0,3], \partial) \times I ; X) .
$$

The evaluation map $\sigma: \Sigma C\left(I^{2} ; X\right) \rightarrow J(\Sigma X)$ defined in this way is filtration-preserving up to homotopy, and so its restrictions give maps

$$
\sigma_{k}: \Sigma C_{k}\left(I^{2} ; X\right) \rightarrow J_{k}(\Sigma X)
$$

inducing the reduced evaluation maps

$$
\bar{\sigma}_{k}: \Sigma D_{k}\left(I^{2} ; X\right)=\Sigma\left(C_{k} / C_{k-1}\right) \rightarrow(\Sigma X)^{\wedge k}=J_{k}(\Sigma X) / J_{k-1}(\Sigma X)
$$


for $k \geq 1$, where $\sigma_{1}=\bar{\sigma}_{1}: \Sigma C_{1}\left(I^{2} ; X\right)=\Sigma X \rightarrow J_{1}(\Sigma X)$ is a homotopy equivalence. Moreover, by applying the naturality of the Hopf invariants on $\left(M, M_{0}\right)$ to the composites in the definition of the evaluation map $\sigma$, we get the following homotopy commutative diagram:

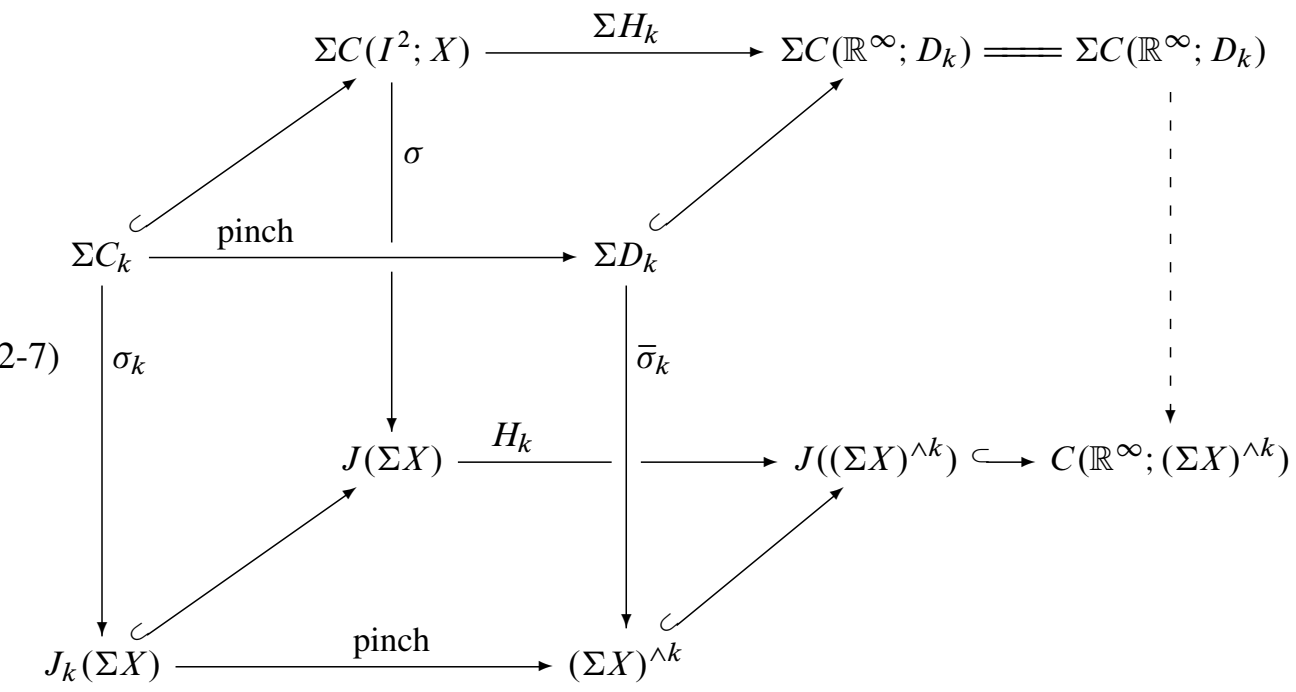

Let $\operatorname{sk}_{n}(Y)$ denote the $n^{\text {th }}$ skeleton of $Y$.

Theorem 2.3 Let $X$ be a simply connected space with connectivity $|X|$ such that $\operatorname{id}_{\Sigma^{2} X}$ has exponent 4 in $\left[\Sigma^{2} X, \Sigma^{2} X\right]$. Then there is a map

$$
\tilde{H}_{2}: \operatorname{sk}_{4|X|-1}\left(\Omega^{2} \Sigma^{2} X\right) \rightarrow D_{2}\left(I^{2} ; X\right)
$$

such that the adjoint map of the $4^{\text {th }}$ power $4: \Omega^{2} \Sigma^{2} X \rightarrow \Omega^{2} \Sigma^{2} X$ restricted to $\mathrm{sk}_{4|X|-1}\left(\Omega^{2} \Sigma^{2} X\right)$ is homotopic to the composite

$$
\Sigma \mathrm{sk}_{4|X|-1}\left(\Omega^{2} \Sigma^{2} X\right) \stackrel{\Sigma \widetilde{H}_{2}}{\longrightarrow} \Sigma D_{2}\left(I^{2} ; X\right) \stackrel{\bar{\sigma}_{2}}{\longrightarrow}(\Sigma X)^{\wedge 2} \stackrel{2 \cdot S_{2}}{\longrightarrow} \Omega \Sigma^{2} X .
$$

Proof Note that $D_{2}\left(I^{2} ; X\right)$ is the $(4|X|-1)$-skeleton of $C\left(\mathbb{R}^{\infty} ; D_{2}\right)$. There is a homotopy commutative diagram

$$
\begin{array}{ccc}
\operatorname{sk}_{4|X|-1}\left(\Omega^{2} \Sigma^{2} X\right) \hookrightarrow C_{3}\left(I^{2} ; X\right) & \longrightarrow \Omega^{2} \Sigma^{2} X \\
\vdots \tilde{H}_{2} & & \\
\vdots & & H_{2} \\
D_{2} \subset & \longrightarrow C\left(\mathbb{R}^{\infty} ; D_{2}\right)
\end{array}
$$

for some map $\widetilde{H}_{2}$. This gives the map $\widetilde{H}_{2}$ in the statement. 
By Proposition 2.2, $\zeta_{3} \circ \sigma_{3}$ is nullhomotopic. Therefore we only need to consider the obstruction $\zeta_{2}$. By diagram (2-7), using the property that $\left|(\Sigma X)^{\wedge 2}\right|=2(|X|+1)$, there is a homotopy commutative diagram:

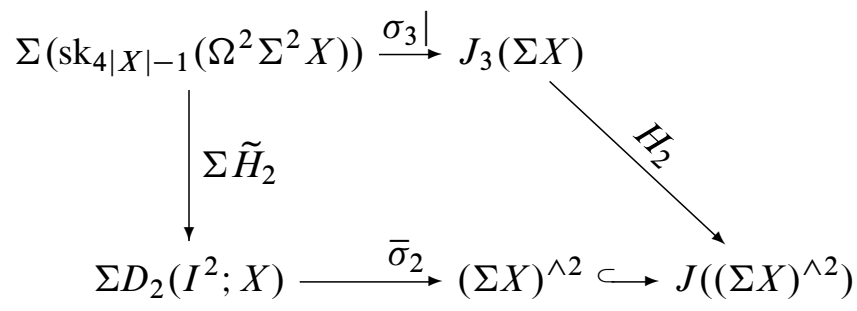

The assertion now follows by Lemma 2.1 .

\section{The reduced evaluation map on mod 2 Moore spaces}

In this section, we give some lemmas on the reduced evaluation map

$$
\bar{\sigma}=\bar{\sigma}_{2}: \Sigma D_{2}=\Sigma D_{2}\left(I^{2} ; X\right)=\Sigma D_{2}\left(\mathbb{R}^{2} ; X\right) \rightarrow(\Sigma X)^{\wedge 2}
$$

in the case that $X$ is a mod 2 Moore space. Let $P^{n}(2)=S^{n-1} \cup_{2} e^{n}$ be the $n-$ dimensional mod 2 Moore space.

Lemma 3.1 [21, Proposition 2.5] Let $n \geq 3$. Then the degree-2 map [2]: $P^{n}(2) \rightarrow$ $P^{n}(2)$ is homotopic to the composite

$$
P^{n}(2) \stackrel{\text { pinch }}{\longrightarrow} S^{n} \stackrel{\eta}{\longrightarrow} S^{n-1} \longrightarrow P^{n}(2) .
$$

Thus the degree-2 map [2]: $P^{n}(2)^{\wedge 2} \rightarrow P^{n}(2)^{\wedge 2}$ is homotopic to the composite

$$
P^{n}(2)^{\wedge 2} \stackrel{\text { pinch }}{\longrightarrow} P^{2 n}(2) \stackrel{\eta \wedge \text { id }}{\longrightarrow} P^{2 n-1}(2) \subset P^{n}(2)^{\wedge 2} .
$$

Let $u, v$ be a basis for the mod 2 homology $\widetilde{H}_{*}\left(P^{n}(2)\right)$ with $|u|=n-1$ and $|v|=n$. Then the mod 2 homology is given by $H_{*}\left(\Omega P^{n+1}(2)\right)=T(u, v)$ with $\mathrm{Sq}_{*}^{1} v=u$. By the work of Dyer and Lashof [11], $\widetilde{H}_{*}\left(D_{2}\left(\mathbb{R}^{2} ; P^{n-1}(2)\right)\right)$ has the following basis:

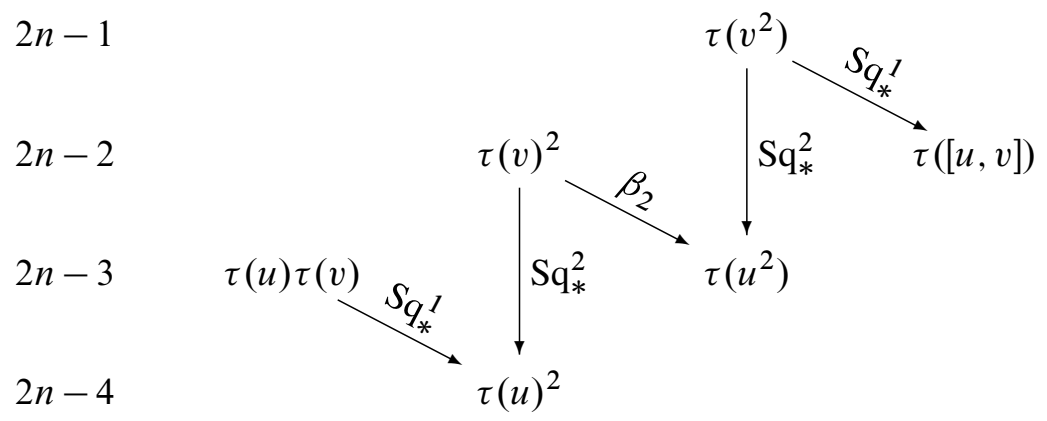


where the Steenrod operations follow from those on $H_{*}\left(\Omega P^{n+1}(2)\right)$.

The reduced evaluation $\bar{\sigma}_{*}: \widetilde{H}_{*}\left(D_{2}\left(\mathbb{R}^{2}, P^{n-1}(2)\right)\right) \rightarrow \widetilde{H}_{*}\left(\left(P^{n}(2)\right)^{\wedge 2}\right)$ is given by

$$
\bar{\sigma}_{*}\left(\tau\left(v^{2}\right)\right)=v^{2}, \quad \bar{\sigma}_{*}(\tau[u, v])=[u, v], \quad \bar{\sigma}_{*}\left(\tau\left(u^{2}\right)\right)=u^{2},
$$

and the three remaining elements are sent to zero.

Let us do cellular analysis on the homotopy of $\Sigma D_{2}\left(\mathbb{R}^{2} ; P^{n-1}(2)\right)$. The elements $\left\{\tau\left(v^{2}\right), \tau([u, v]), \tau(v)^{2}, \tau\left(u^{2}\right)\right\}$ have a structure of $P^{2 n-2}(4)$ attached by $2-$ cells via a map $P^{2 n-2}(2) \rightarrow P^{2 n-2}(4)$.

Let $g_{2}$ be the composite

$$
P^{2 n-2}(2) \stackrel{q}{\longrightarrow} S^{2 n-2} \stackrel{\eta}{\longrightarrow} S^{2 n-3} \hookrightarrow P^{2 n-2}(4) .
$$

Lemma 3.2 There exists a unique 4-cell complex $C^{2 n-1}$ such that mod 2 homology $\tilde{H}_{*}\left(C^{2 n-1}\right)$ has a basis $\left\{a_{2 n-3}, b_{2 n-2}, c_{2 n-2}, d_{2 n-1}\right\}$, with

$$
\beta_{2}(b)=a, \quad \operatorname{Sq}_{*}^{2}(d)=a, \quad \operatorname{Sq}_{*}^{1}(d)=c .
$$

Proof Consider the short exact sequence

$2 \cdot \pi_{2 n-3}\left(P^{2 n-2}(4)\right)=\mathbb{Z} / 2 \nleftarrow\left[P^{2 n-2}(2), P^{2 n-2}(4)\right] \hookleftarrow \pi_{2 n-2}\left(P^{2 n-2}(4)\right)=\mathbb{Z} / 2$.

Let $g_{1}$ be the map in the following commutative diagram of cofibre sequences:

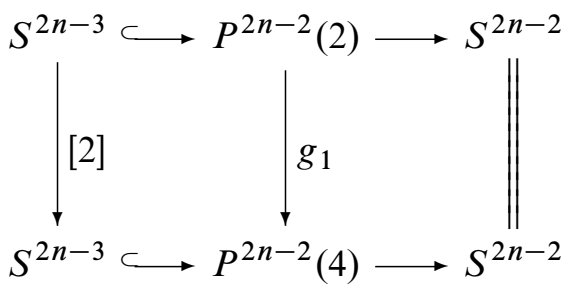

Then $2\left[g_{1}\right]$ is given by the composite

$$
P^{2 n-2}(2) \longrightarrow S^{2 n-2} \stackrel{\eta}{\longrightarrow} S^{2 n-3} \longrightarrow P^{2 n-2}(2) \stackrel{g_{1}}{\longrightarrow} P^{2 n-2}(4),
$$

which is nullhomotopic because $g_{1} \mid S^{2 n-3}$ factors through the degree map [2]: $S^{2 n-3} \rightarrow$ $S^{2 n-3}$. Then

$$
\left[P^{2 n-2}(2), P^{2 n-2}(4)\right]=\mathbb{Z} / 2 \oplus \mathbb{Z} / 2 .
$$

The three essential elements in $\left[P^{2 n-2}(2), P^{2 n-2}(4)\right]$ are given by $\left[g_{1}\right],\left[g_{2}\right],\left[g_{1}+g_{2}\right]$. Since $\left[g_{1}\right]_{*},\left[g_{1}+g_{2}\right]_{*}: H_{2 n-2}\left(P^{2 n-2}(2)\right) \rightarrow H_{2 n-2}\left(P^{2 n-2}(4)\right)$ are nonzero, $\left[g_{2}\right]$ is the only homotopy class as the attaching map for $C^{2 n-1}$. The proof is finished. 
By pinching out the bottom Moore space, we have a pinch map

$$
\phi: \Sigma D_{2}\left(\mathbb{R}^{2} ; P^{n-1}(2)\right) \rightarrow C^{2 n}
$$

with a commutative diagram

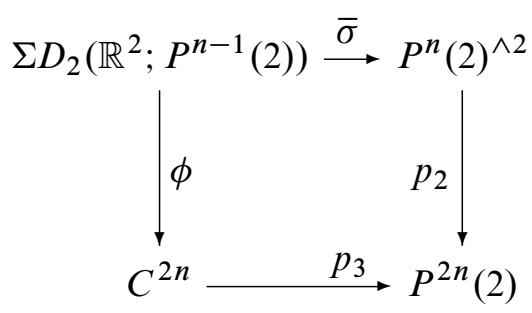

where $p_{3}$ induces an epimorphism on mod 2 homology.

Let $\widetilde{C}^{2 n-1}$ and $\bar{C}^{2 n-1}$ be defined in the commutative diagram

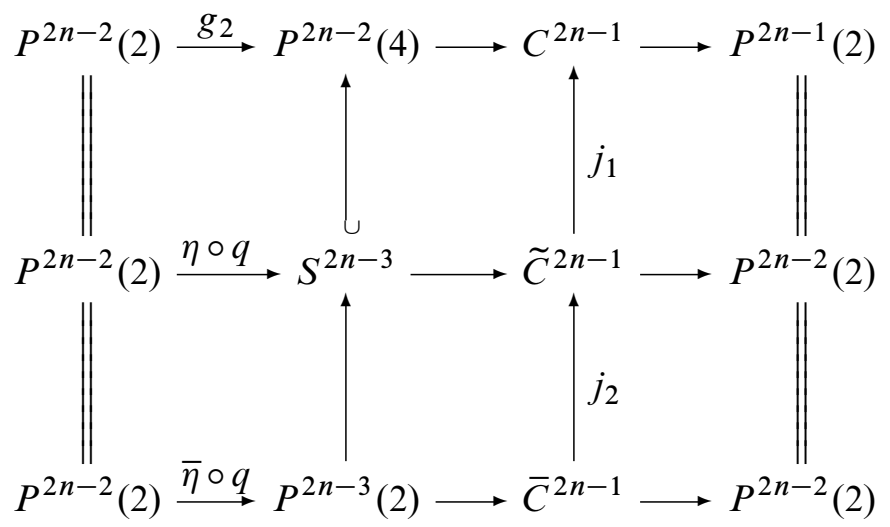

where $\bar{\eta}: S^{2 n-2} \rightarrow P^{2 n-3}(2)$ is a lifting of $\eta, g_{2}$ is defined by (3-1) and the rows are cofibre sequences. Observe that:

(1) The map $j_{1}$ induces a monomorphism on mod 2 homology.

(2) The homotopy cofibre of $j_{1} \circ j_{2}: \bar{C}^{2 n-1} \rightarrow C^{2 n-1}$ is the same as the homotopy cofibre of $P^{2 n-3}(2) \rightarrow S^{2 n-3} \rightarrow P^{2 n-2}(4)$.

(3) The mod 2 homology $\widetilde{H}_{*}\left(\bar{C}^{2 n-1}\right)$ has a basis $\left\{x_{2 n-1}, x_{2 n-2}, x_{2 n-3}, x_{2 n-4}\right\}$, with

$$
\begin{aligned}
\mathrm{Sq}_{*}^{1}\left(x_{2 n-1}\right) Y & =x_{2 n-2}, \quad \mathrm{Sq}_{*}^{1}\left(x_{2 n-3}\right)=x_{2 n-4} \\
\mathrm{Sq}_{*}^{2}\left(x_{2 n-1}\right) & =x_{2 n-3}, \quad \mathrm{Sq}_{*}^{2}\left(x_{2 n-2}\right)=x_{2 n-4}
\end{aligned}
$$

Lemma 3.3 The homotopy cofibre of $P^{2 n-3}(2) \rightarrow S^{2 n-3} \rightarrow P^{2 n-2}(4)$ is $P^{2 n-2}(8)$. 
Proof This follows from the commutative diagram of cofibre sequences:

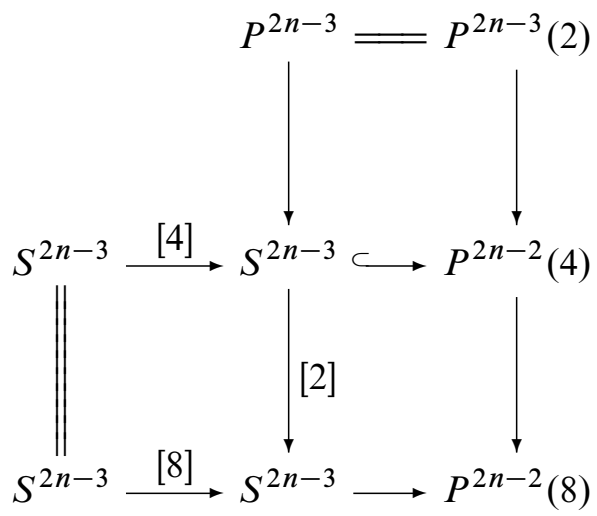

Lemma 3.4 The space $\bar{C}^{2 n-1}$ is homotopy equivalent to $\Sigma^{2 n-7} \mathbb{C} \mathrm{P}^{2} \wedge \mathbb{R} \mathrm{P}^{2}$.

Proof We only need to show that there is a unique 4-cell complex having the same homology as $\bar{C}^{2 n+1}$ with the same Steenrod module structure.

Consider the homotopy classes $\left[P^{2 n-2}(2), P^{2 n-3}(2)\right]$. There is a short exact sequence

$$
\pi_{2 n-3}\left(P^{2 n-3}(2)\right)=\mathbb{Z} / 2 \longleftarrow\left[P^{2 n-2}(2), P^{2 n-3}(2)\right] \hookleftarrow \pi_{2 n-2}\left(P^{2 n-3}(2)\right) / 2 .
$$

There are three essential homotopy classes in $\left[P^{2 n-2}(2), P^{2 n-3}(2)\right]$ given as follows:

(1) $\eta \wedge$ id: $P^{2 n-2}(2) \rightarrow P^{2 n-3}(2)$. The homology of its cofibre has the same structure as $\tilde{H}_{*}\left(\Sigma^{2 n-7} \mathbb{C} \mathrm{P}^{2} \wedge \mathbb{R P}^{2}\right)$.

(2) The composite $h_{1}: P^{2 n-2}(2) \stackrel{\text { pinch }}{\longrightarrow} S^{2 n-2} \stackrel{\bar{\eta}}{\longrightarrow} P^{2 n-3}(2)$. The reduced mod 2 homology of the cofibre $C_{h_{1}}$ has a basis

$$
\left\{y_{2 n-1}, y_{2 n-2}, y_{2 n-3}, y_{2 n-4}\right\}
$$

with

$$
\begin{array}{ll}
\mathrm{Sq}_{*}^{1}\left(y_{2 n-1}\right)=y_{2 n-2}, & \mathrm{Sq}_{*}^{1}\left(y_{2 n-3}\right)=y_{2 n-4}, \\
\mathrm{Sq}_{*}^{2}\left(y_{2 n-1}\right)=y_{2 n-3}, & \operatorname{Sq}_{*}^{2}\left(y_{2 n-2}\right)=0 .
\end{array}
$$

Here $\operatorname{Sq}_{*}^{2}\left(y_{2 n-2}\right)=0$ because $\left.h_{1}\right|_{S^{2 n-3}}$ is nullhomotopic.

(3) The composite $h_{2}: P^{2 n-2}(2) \stackrel{\tilde{\eta}}{\longrightarrow} S^{2 n-4} \longrightarrow P^{2 n-3}(2)$, where $\tilde{\eta}$ is an extension of $\eta: S^{2 n-3} \rightarrow S^{2 n-4}$. The reduced mod 2 homology of the cofibre $C_{h_{2}}$ has a basis

$$
\left\{z_{2 n-1}, z_{2 n-2}, z_{2 n-3}, z_{2 n-4}\right\}
$$


with

$$
\begin{array}{ll}
\mathrm{Sq}_{*}^{1}\left(z_{2 n-1}\right)=z_{2 n-2}, & \mathrm{Sq}_{*}^{1}\left(z_{2 n-3}\right)=z_{2 n-4}, \\
\mathrm{Sq}_{*}^{2}\left(y_{2 n-2}\right)=y_{2 n-4}, & \operatorname{Sq}_{*}^{2}\left(y_{2 n-1}\right)=0 .
\end{array}
$$

Here $\operatorname{Sq}_{*}^{2}\left(y_{2 n-2}\right)=0$ because

$$
P^{2 n-2}(2) \stackrel{h_{2}}{\longrightarrow} P^{2 n-3}(2) \rightarrow S^{2 n-3}
$$

is nullhomotopic.

The proof is finished.

The following lemma will be useful.

Lemma 3.5 There is a commutative diagram

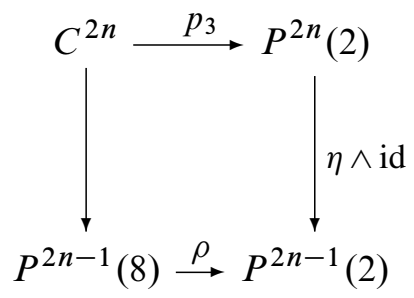

where $\rho_{*}: H_{2 n-2}\left(P^{2 n-1}(8) ; \mathbb{Z} / 2\right) \rightarrow H_{2 n-2}\left(P^{2 n-2}(2) ; \mathbb{Z} / 2\right)$ is an isomorphism.

Proof From property (2) of diagram (3-3) and Lemma 3.4, there is a commutative diagram of cofibre sequences:

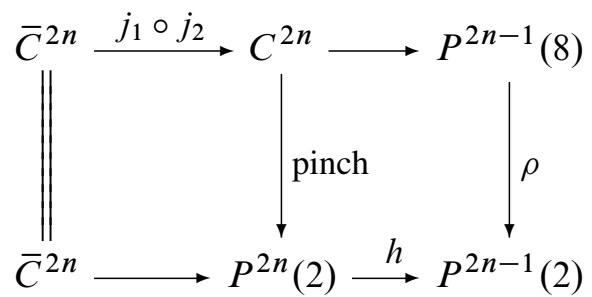

By Lemma 3.4, $h \simeq \eta \wedge$ id, and hence the result follows.

Let us first prove the special case of Theorem 1.1 when the Whitehead square is divisible by 2 .

Theorem 3.6 Let $n+1 \equiv 0 \bmod 4$ with $n+1>5$. Suppose that the Whitehead square $\omega_{n}$ is divisible by 2 . Then the $4^{\text {th }}$ power map $4 \mid: \Omega^{2} P^{n+1}(2) \rightarrow \Omega^{2} P^{n+1}(2)$ restricted to the skeleton $\mathrm{sk}_{4(n-1)-1}$ is nullhomotopic. 
Proof By Theorem 2.3, it suffices to show that the composite

$$
\Sigma D_{2} \stackrel{\bar{\sigma}_{2}}{\longrightarrow}\left(P^{n}(2)\right)^{\wedge 2} \stackrel{[2]}{\longrightarrow}\left(P^{n}(2)\right)^{\wedge 2} \stackrel{S_{2}}{\longrightarrow} \Omega P^{n+1}(2)
$$

is nullhomotopic. By Lemma 3.1, it suffices to prove that the composite

$$
\Sigma D_{2} \stackrel{\bar{\sigma}_{2}}{\longrightarrow}\left(P^{n}(2)\right)^{\wedge 2} \stackrel{\text { pinch }}{\longrightarrow} P^{2 n}(2) \stackrel{\eta \wedge \text { id }}{\longrightarrow} P^{2 n-1}(2) \stackrel{S_{2} \mid}{\longrightarrow} \Omega P^{n+1}(2)
$$

is nullhomotopic.

Since $\omega_{n}$ is divisible by 2 ,

$$
\left.S_{2}\right|_{S^{2 n-2}}: S^{2 n-2} \rightarrow \Omega P^{n+1}(2)
$$

is nullhomotopic. Thus $S_{2} \mid: P^{2 n-1}(2) \rightarrow \Omega P^{n+1}(2)$ factors through $S^{2 n-1}$.

Let $\rho: P^{2 n-1}(8) \rightarrow P^{2 n-1}(2)$ be the map in Lemma 3.5. We show that there is a commutative diagram of cofibre sequences:

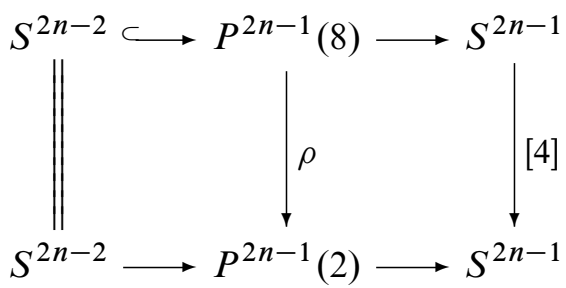

There is a short exact sequence

$$
\pi_{2 n-2}\left(P^{2 n-1}(2)\right)=\mathbb{Z} / 2 \longleftarrow\left[P^{2 n-1}(8), P^{2 n-1}(2)\right] \hookleftarrow \pi_{2 n-1}\left(P^{2 n-1}(2)\right)=\mathbb{Z} / 2 .
$$

Let $\alpha: P^{2 n-1}(8) \rightarrow P^{2 n-1}(2)$ be an extension of the inclusion map $S^{2 n-2} \rightarrow P^{2 n-1}(2)$. Then $2[\alpha]=0$. Thus

$$
\left[P^{2 n-1}(8), P^{2 n-1}(2)\right]=\mathbb{Z} / 2 \oplus \mathbb{Z} / 2 .
$$

Let $\beta$ be the composite

$$
P^{2 n-1}(8) \stackrel{\text { pinch }}{\longrightarrow} S^{2 n-1} \stackrel{\eta}{\longrightarrow} S^{2 n-2} \longrightarrow P^{2 n-2}(2) .
$$

Then $\left[P^{2 n-1}(8), P^{2 n-1}(2)\right]$ is generated by $\alpha$ and $\beta$. We can make a choice of $\alpha$ from the commutative diagram:

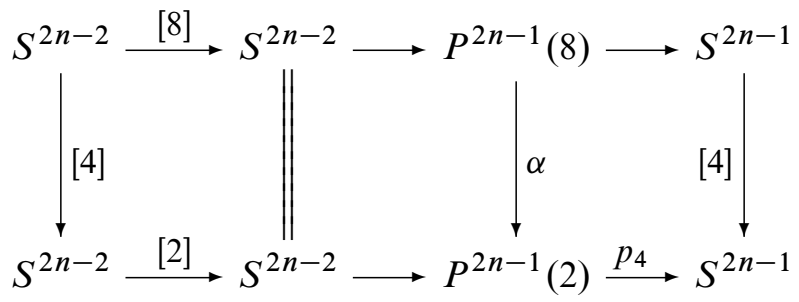


Then $[\rho]$ equals $[\alpha]$ or $[\alpha+\beta]$. Since $p_{4 *}[\beta]=0$, diagram (3-5) holds.

Now the assertion follows from the commutative diagram

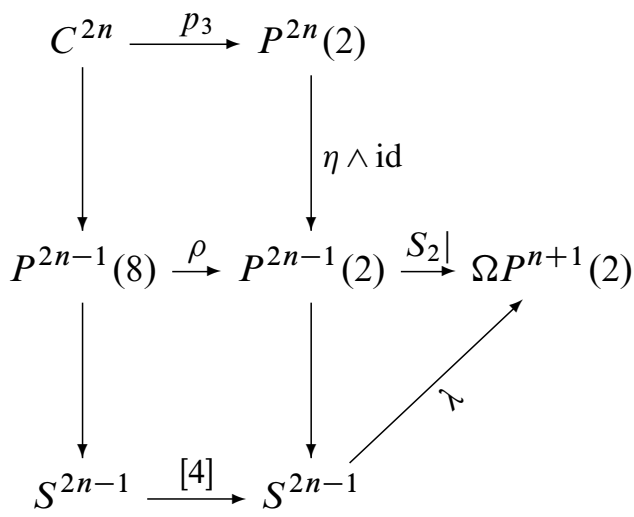

which is obtained from Lemma 3.5 and diagram (3-5), together with diagram (3-2) and the fact [10] that $[\lambda] \in \pi_{2 n}\left(P^{n+1}(2)\right)$ is of order 4 when $n+1 \equiv 0 \bmod 4$.

\section{Some lemmas on $P^{2 n}(2)$}

In this section, we give some lemmas related to the Whitehead products.

Lemma 4.1 Let $j_{2 n+1}$ be the composite

$$
\mathbb{R P}^{2 n} \hookrightarrow \mathrm{SO}(2 n+1) \longrightarrow \Omega^{2 n+1} S^{2 n+1} .
$$

Then

$$
\Omega^{2 n+1}([2]) \circ j_{2 n+1} \simeq 2 \circ j_{2 n+1} .
$$

Proof By [7, Proposition 4.3], the maps $\Omega$ [2], 2: $\Omega S^{2 n+1} \rightarrow \Omega S^{2 n+1}$ differ by the homotopy class represented by the composite

$$
\Omega S^{2 n+1} \stackrel{H_{2}}{\longrightarrow} \Omega S^{4 n+1} \stackrel{\Omega \omega_{2 n+1}}{\longrightarrow} \Omega S^{2 n+1} .
$$

From the commutative diagram

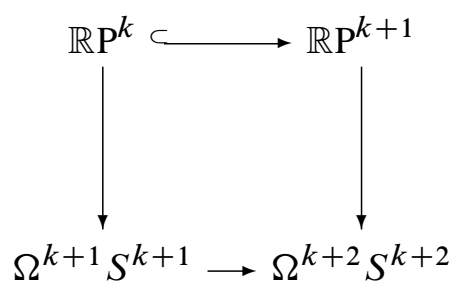


there is a commutative diagram

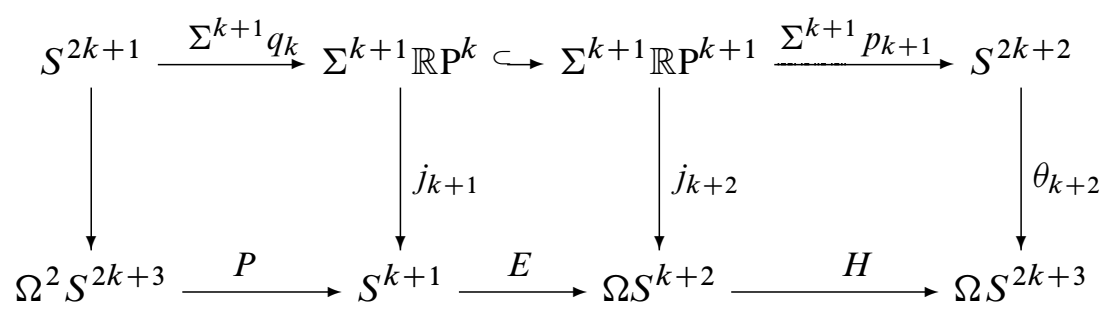

where $q_{k}: S^{k} \rightarrow \mathbb{R} \mathrm{P}^{k}$ is the projection map, $p_{k+1}: \mathbb{R} \mathrm{P}^{k+1} \rightarrow S^{k+1}$ is the pinch map, the top row is a cofibre sequence, and the bottom row is the EHP sequence. The map

$$
\theta_{2 k+2}: H_{2 k+2}\left(S^{2 k+2}\right) \rightarrow H_{2 k+2}\left(\Omega S^{2 k+3}\right)
$$

is an isomorphism [13, Theorem (1.1)]. It follows that there is a commutative diagram:

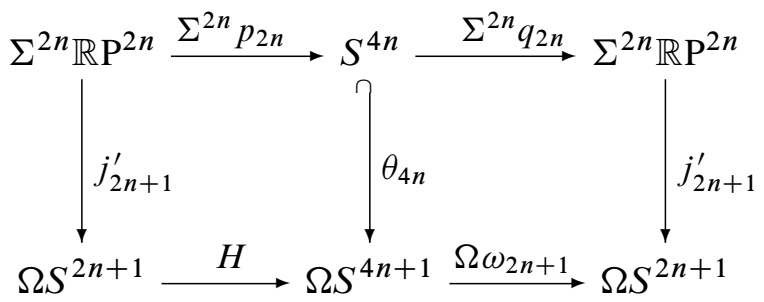

We check that the composite

$$
\Sigma^{2} \mathbb{R} \mathrm{P}^{2 n} \stackrel{\Sigma^{2} p_{2 n}}{\longrightarrow} S^{2 n+2} \stackrel{\Sigma^{2} q_{2 n}}{\longrightarrow} \Sigma^{2} \mathbb{R} \mathrm{P}^{2 n}
$$

is nullhomotopic; the assertion will then follow from the above commutative diagram.

Consider the Hopf map

$$
H: \Sigma \mathbb{R} \mathrm{P}^{\infty} \wedge \mathbb{R} \mathrm{P}^{\infty} \rightarrow \Sigma \mathbb{R} \mathrm{P}^{\infty}
$$

We can see that the composite

$$
\Sigma \mathbb{R} \mathrm{P}^{2 n} \wedge \mathbb{R} \mathrm{P}^{1} \hookrightarrow \Sigma \mathbb{R} \mathrm{P}^{\infty} \wedge \mathbb{R} \mathrm{P}^{\infty} \stackrel{H}{\longrightarrow} \Sigma \mathbb{R} \mathrm{P}^{\infty}
$$

maps into $\Sigma \mathbb{R} \mathrm{P}^{2 n+1}$ by considering the skeleton. Let

$$
f: \Sigma^{2} \mathbb{R} \mathrm{P}^{2 n}=\Sigma \mathbb{R} \mathrm{P}^{2 n} \wedge \mathbb{R} \mathrm{P}^{1} \rightarrow \Sigma \mathbb{R} \mathrm{P}^{2 n+1}
$$

be the resulting map. Recall that the mod 2 homology $\left.H_{*}(\mathbb{R P})^{\infty}\right)=\Gamma(u)$ with $|u|=1$ is the divided power algebra. The Hopf map $H$ induces

$$
H_{*}\left(\Sigma\left(\gamma_{2 n}(u) \otimes \gamma_{1}(u)\right)\right)=\Sigma \gamma_{2 n+1}(u) .
$$

Thus

$$
f_{*}: H_{2 n+2}\left(\Sigma^{2} \mathbb{R} \mathrm{P}^{2 n} ; \mathbb{Z} / 2\right) \rightarrow H_{2 n+2}\left(\Sigma \mathbb{R} \mathrm{P}^{2 n+1} ; \mathbb{Z} / 2\right)
$$


is an isomorphism. It follows that the pinch map $\Sigma^{2} p_{2 n}: \Sigma^{2} \mathbb{R} \mathrm{P}^{2 n} \rightarrow S^{2 n+2}$ factors through $f$. From the cofibre sequence

$$
\Sigma \mathbb{R} \mathrm{P}^{2 n+1} \stackrel{\Sigma p_{2 n+1}}{\longrightarrow} S^{2 n+2} \stackrel{\Sigma^{2} q_{2 n}}{\longrightarrow} \Sigma^{2} \mathbb{R} \mathrm{P}^{2 n}
$$

we obtain that $\Sigma^{2}\left(q_{2 n} \circ p_{2 n}\right)$ is nullhomotopic.

Lemma 4.2 There is a homotopy commutative diagram

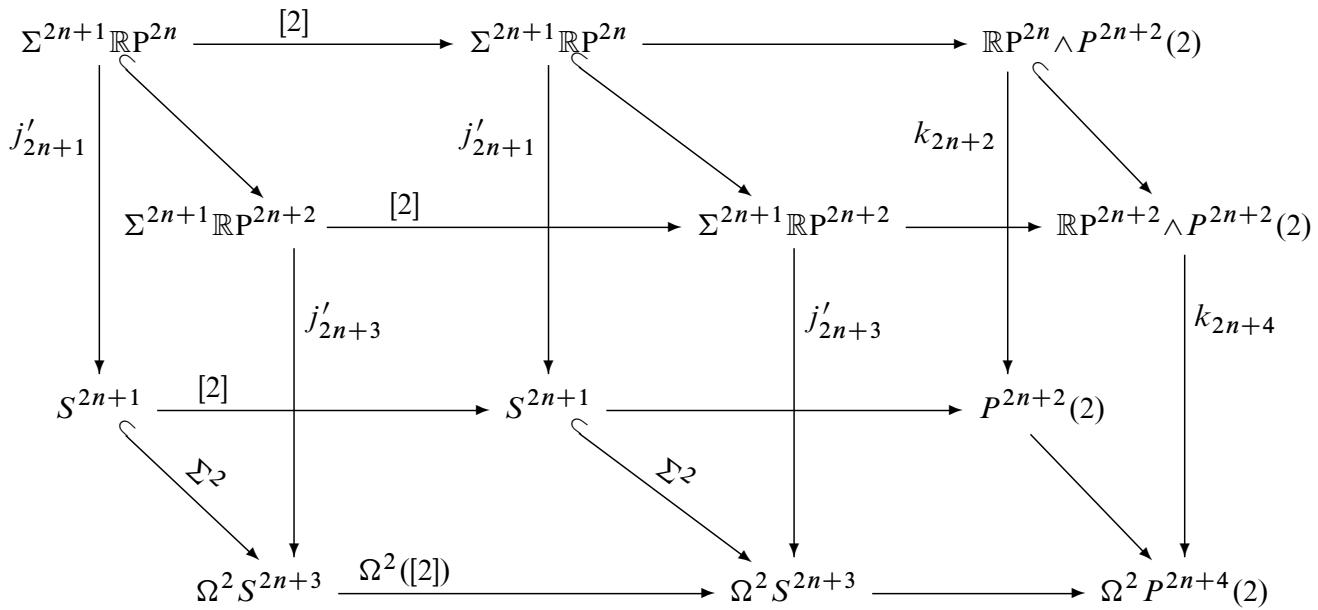

Proof By Lemma 4.1, the left cube is homotopy commutative. Here the morphism from the back face to the front face in the left cube is given by inclusions and all faces except the back and the front commute strictly. The homotopy for the front face of the left cube restricts to the homotopy for the rear face of the left cube. We can replace the four objects in the right face of the left cube with the mapping cylinders and replace the four rightward-pointing arrows in the left cube with inclusions. The homotopy extension property implies that there is a choice of two downward arrows in the right face of the new left cube that make it commutative. Taking the cofibres of the right arrows in the new left cube and using the property that the composition of the bottom two arrows is nullhomotopic, we conclude that there exists a map

$$
k_{2 n+2}: \mathbb{R} \mathrm{P}^{2 n} \wedge P^{2 n+2}(2) \rightarrow P^{2 n+2}(2)
$$

such that the right cube in the diagram commutes up to homotopy.

Mahowald has a result $[14$, Theorem (1.1.2a) $]$ that $\left[\iota_{4 n-1}, \eta_{4 n-1}\right]=0$. For $\bmod 2$ Moore spaces, we have the following lemma. 
Lemma 4.3 There exists a map $\delta_{4 n}: P^{8 n-2}(2) \rightarrow P^{4 n}(2)$ with the following properties:

(1) There is a homotopy commutative diagram:

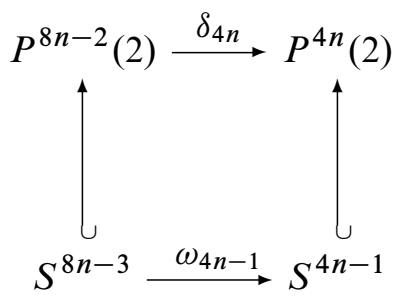

(2) The composite

$$
P^{8 n-2}(2) \stackrel{\delta_{4 n}}{\longrightarrow} P^{4 n}(2) \stackrel{\Sigma^{2}}{\longrightarrow} \Omega^{2} P^{4 n+2}(2)
$$

is nullhomotopic.

(3) The composite

$$
P^{8 n-1}(2) \stackrel{\eta \wedge \mathrm{id}}{\longrightarrow} P^{8 n-2}(2) \stackrel{\delta_{4 n}}{\longrightarrow} P^{4 n}(2)
$$

is nullhomotopic.

Proof Let $W_{k}^{n}$ be the homotopy of the homotopy fibre of the inclusion map $S^{n} \rightarrow$ $\Omega^{k} S^{n+k}$, and let $W_{k}^{n}(2)$ be the homotopy fibre of the inclusion map $P^{n}(2) \rightarrow$ $\Omega^{k} P^{n+k}(2)$. By Lemma 3.2, there is a homotopy commutative diagram

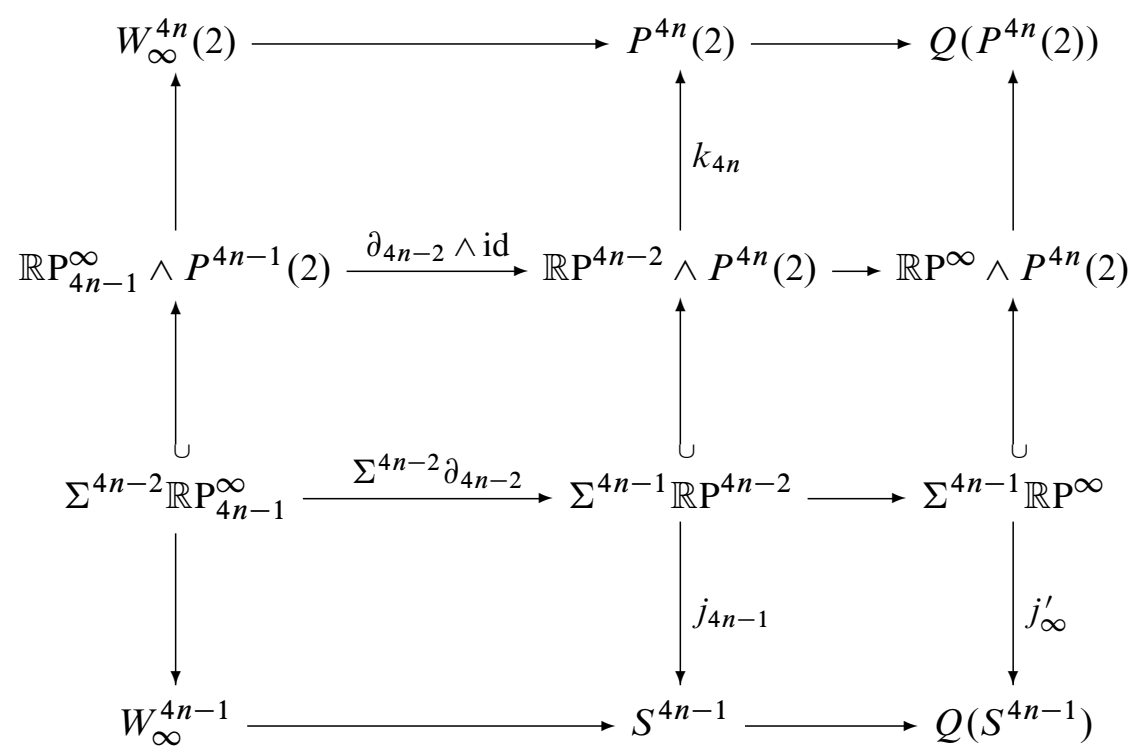


with a canonical morphism of fibre sequences from the bottom row to the top row for making a homotopy commutative diagram of cubic diagrams, where

$$
\partial_{k}: \mathbb{R} \mathrm{P}_{k+1}^{\infty}=\mathbb{R} \mathrm{P}^{\infty} / \mathbb{R} \mathrm{P}^{k} \rightarrow \Sigma \mathbb{R} \mathrm{P}^{k}
$$

is the boundary map and $Q(X)=\Omega^{\infty} \Sigma^{\infty} X$. Let

$$
\delta_{4 n}: P^{8 n-2}(2) \rightarrow P^{4 n}(2)
$$

be the composite

$S^{4 n-1} \wedge P^{4 n-1}(2) \hookrightarrow \mathbb{R P}_{4 n-1}^{\infty} \wedge P^{4 n-1}(2) \stackrel{\partial_{4 n-2} \wedge \mathrm{id}}{\longrightarrow} \mathbb{R} \mathrm{P}^{4 n-2} \wedge P^{4 n}(2) \stackrel{k_{4 n}}{\longrightarrow} P^{4 n}(2)$.

From the above commutative diagram, $\delta_{4 n} \mid: S^{8 n-3} \rightarrow P^{4 n}(2)$ is homotopic to the composite

$$
S^{8 n-3} \stackrel{\omega_{4 n-1}}{\longrightarrow} S^{4 n-1} \subset P^{4 n}(2)
$$

Moreover, the composite

$$
P^{8 n-2}(2) \stackrel{\delta_{4 n}}{\longrightarrow} P^{4 n}(2) \longrightarrow Q\left(P^{4 n}(2)\right)
$$

is nullhomotopic by construction. It follows that $\Sigma^{2} \delta_{4 n}: P^{8 n}(2) \rightarrow P^{4 n+2}(2)$ is nullhomotopic for dimensional reasons.

Now we check condition (3) in the statement. Observe that the reduced mod 2 homology of $\mathbb{R P}_{4 n-1}^{\infty}$ has a basis $\left\{u^{k}\right\}$ with $k \geq 4 n-1$. The Steenrod operation satisfies

$$
\operatorname{Sq}^{2}\left(u^{4 k-1}\right)=\operatorname{Sq}^{2}\left(u^{4 k-4} \cdot u^{3}\right)=u^{4 k-4} \operatorname{Sq}^{2}\left(u^{3}\right)=u^{4 k+1}
$$

Thus

$$
\mathrm{Sq}_{*}^{2}: H_{8 n-1}\left(\Sigma^{4 n-2} \mathbb{R} \mathrm{P}_{4 n-1}^{\infty}\right)=\mathbb{Z} / 2 \rightarrow H_{8 n-3}\left(\Sigma^{4 n-2} \mathbb{R} \mathrm{P}_{4 n-1}^{\infty}\right)=\mathbb{Z} / 2
$$

is an isomorphism. It follows that the composite

$$
S^{8 n-2} \stackrel{\eta}{\longrightarrow} S^{8 n-3} \subset \Sigma^{4 n-2} \mathbb{R P}_{4 n-1}^{\infty}
$$

is nullhomotopic. By smashing with mod 2 Moore spaces, the composite

$$
P^{8 n-1}(2) \stackrel{\eta \wedge \mathrm{id}}{\longrightarrow} P^{8 n-2}(2) \subset \Sigma^{4 n-2} \mathbb{R} \mathrm{P}_{4 n-1}^{\infty} \wedge P^{4 n-1}(2)
$$

is nullhomotopic. Condition (3) is satisfied and hence the result follows. 


\section{Proof of Theorem 1.1}

We use the notation $W_{k}^{n}(2)$ defined in the proof of Lemma 4.3. Consider the homotopy commutative diagram of fibre sequences:

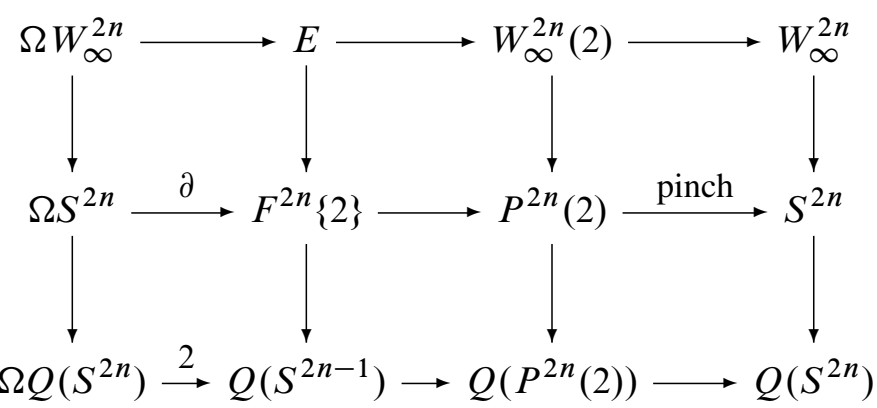

For a space $X$, let $\left\{P^{n}(2), X\right\}=\left[P^{n}(2), Q(X)\right]$ denote the group of stable homotopy classes from $P^{n}(2)$ to $X$.

Lemma 5.1 (1) The stabilisation $\left[P^{4 n-2}(2), S^{2 n}\right] \rightarrow\left\{P^{4 n-2}(2), S^{2 n}\right\}$ is an isomorphism.

(2) The stabilisation $\left[P^{4 n-2}(2), \Omega S^{2 n}\right] \rightarrow\left[P^{4 n-2}(2), \Omega Q\left(S^{2 n}\right)\right]$ is onto.

(3) Let $4 n \neq 4,8$. Then the kernel of $\left[P^{8 n-2}(2), S^{4 n-1}\right] \rightarrow\left\{P^{8 n-2}(2), S^{4 n-1}\right\}$ is $\mathbb{Z} / 2$, generated by any map $\phi: P^{8 n-2}(2) \rightarrow S^{4 n-1}$ such that $\left.\phi\right|_{S^{8 n-3}}$ is the Whitehead square.

Proof Assertions (1) and (2) follows immediately from the fact that $S^{2 n}$ is the (4n-1)skeleton of $\Omega S^{2 n+1}$. For assertion (3), consider homotopy commutative diagram of fibre sequences:

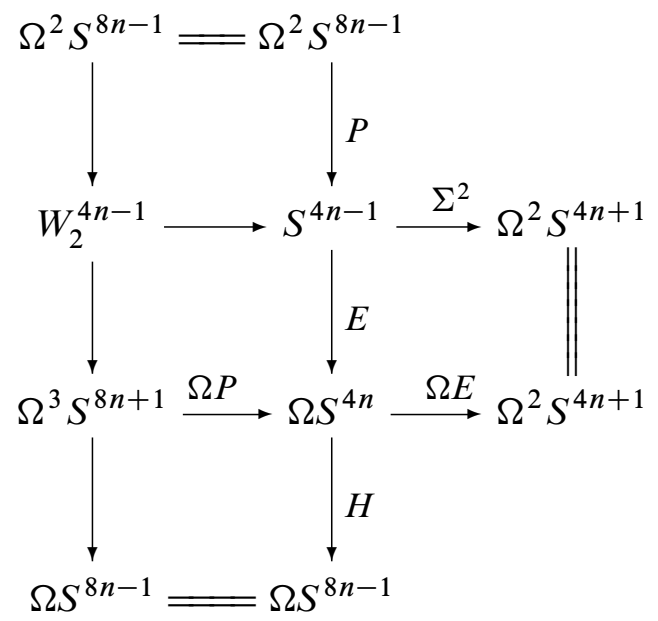


Since the composite

$$
S^{8 n-2} \hookrightarrow \Omega^{3} S^{8 n+1} \stackrel{\Omega P}{\longrightarrow} \Omega S^{4 n} \stackrel{H}{\longrightarrow} \Omega S^{8 n-1}
$$

is of degree 2, we have

$$
\operatorname{sk}_{8 n-1}\left(W_{2}^{4 n-1}\right)=P^{8 n-2}(2)
$$

It follows that there is an exact sequence

$$
\begin{aligned}
{\left[P^{8 n-2}(2), \Omega^{3} S^{4 n+1}\right] \rightarrow\left[P^{8 n-2}(2), P^{8 n-2}(2)\right] } & \\
& \rightarrow\left[P^{8 n-2}(2), S^{4 n-1}\right] \rightarrow\left\{P^{8 n-2}(2), S^{4 n-1}\right\} .
\end{aligned}
$$

By the proof of Lemma 4.3, $\omega_{4 n-1} \circ \eta$ is nullhomotopic. Thus the composite

$$
P^{8 n-2}(2) \longrightarrow S^{8 n-2} \stackrel{\eta}{\longrightarrow} S^{8 n-3} \longrightarrow P^{8 n-2}(2) \longrightarrow S^{4 n-1}
$$

is nullhomotopic, and so the image of

$$
\left[P^{8 n-2}(2), P^{8 n-2}(2)\right]=\mathbb{Z} / 4
$$

in $\left[P^{8 n-4}(2), S^{4 n-1}\right]$ is $\mathbb{Z} / 2$. The proof is finished.

Lemma 5.2 There is a homotopy decomposition

$$
\Omega F^{2 n}\{2\} \simeq \Omega S^{2 n-1} \times \Omega S^{4 n-2} \times \Omega P^{6 n-3}(2)
$$

up to dimension $8 n-8$.

Proof Consider the homotopy commutative diagram of fibre sequences

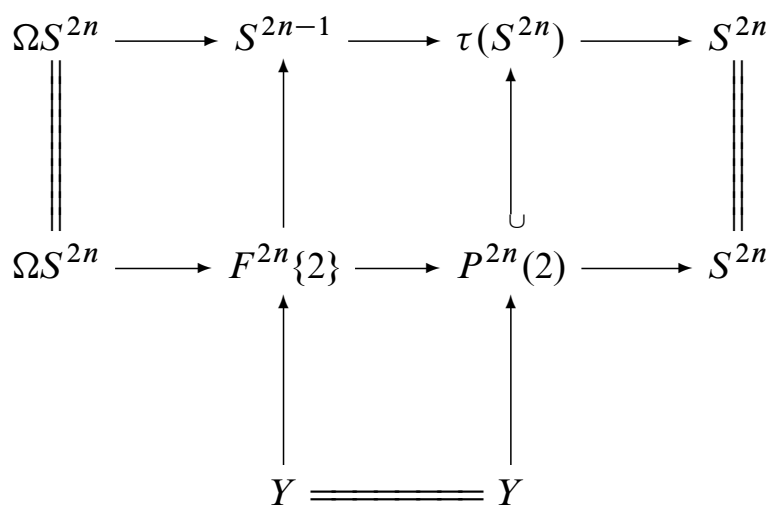

where $\tau\left(S^{2 n}\right)=\mathrm{SO}(2 n+1) / \mathrm{SO}(2 n-1)=V_{2,2 n+1}$ is the 2 -frame Stiefel manifold. Since the map $F^{2 n}\{2\} \rightarrow S^{2 n-1}$ admits a cross-section, there is a homotopy decomposition

$$
\Omega F^{2 n}\{2\} \simeq \Omega Y \times \Omega S^{2 n-1} .
$$


Let $f: \Omega S^{4 n-2} \rightarrow \Omega Y$ be the extension of the inclusion of the bottom cell. Let $g: \Omega P^{6 n-3}(2)=\Omega \Sigma L_{3}\left(P^{2 n-1}(2)\right) \rightarrow \Omega Y$ be the map in the functorial decomposition of

$$
\Omega \Sigma X \simeq \Omega \Sigma L_{3}(X) \times ?
$$

for 2-local spaces $[10 ; 20]$. Then the map

$$
\Omega S^{4 n-2} \times \Omega P^{6 n-3}(2) \stackrel{(f, g)}{\longrightarrow} \Omega Y
$$

induces an isomorphism on homology up to dimension $8 n-8$. The proof is finished.

We restate Theorem 1.1 as follows.

Theorem 5.3 Let $n>1$. The power map 4: $\Omega^{2} P^{4 n}(2) \rightarrow \Omega^{2} P^{4 n}(2)$ restricted to the skeleton $\mathrm{sk}_{4(4 n-2)-1}\left(\Omega^{2} P^{4 n}(2)\right)$ is nullhomotopic.

Proof If the Whitehead square $\omega_{4 n-1}$ is divisible by 2 , we have proved the assertion in Theorem 3.6. Now we assume that $\omega_{4 n-1}$ is not divisible by 2. Similar to the situation in the proof of Theorem 3.6, it suffices to prove that the composite

$$
\Sigma D_{2} \stackrel{\bar{\sigma}_{2}}{\longrightarrow}\left(P^{4 n-1}(2)\right)^{\wedge 2} \stackrel{\text { pinch }}{\longrightarrow} P^{8 n-2}(2)
$$

(2) $\stackrel{\eta \wedge \text { id }}{\longrightarrow} P^{8 n-3}$

(2) $\stackrel{S_{2} \mid}{\longrightarrow} \Omega P^{4 n}$

is nullhomotopic. Our proof is given by controlling the map

$$
S_{2} \mid: P^{8 n-1}(2) \rightarrow \Omega P^{4 n}(2) .
$$

By Lemma 5.2, $\Omega F^{4 n}\{2\} \simeq \Omega S^{4 n-1} \times \Omega S^{8 n-2}$ up to dimension $15 n-5$. Thus

$$
\begin{aligned}
{\left[P^{8 n-2}(2), F^{4 n}\{2\}\right] } & \cong\left[P^{8 n-2}(2), S^{4 n-1}\right] \oplus\left[P^{8 n-2}(2), S^{8 n-2}\right] \\
& =\left[P^{8 n-2}(2), S^{4 n-1}\right] \oplus \mathbb{Z} / 2 .
\end{aligned}
$$

By Lemma 5.1(3), there is an exact sequence

$$
\mathbb{Z} / 2 \rightarrow\left[P^{8 n-2}(2), S^{4 n-1}\right] \rightarrow\left\{P^{8 n-2}(2), S^{4 n-1}\right\} .
$$

Consider the homotopy commutative diagram (5-1) with replacing $2 n$ by $4 n$. Let $g: P^{8 n-2}(2) \rightarrow P^{4 n}(2)$ be a map representing an element in

$$
\operatorname{Ker}\left(\left[P^{8 n-2}(2), P^{4 n}(2)\right] \rightarrow\left\{P^{8 n-2}(2), P^{4 n}(2)\right\}\right) .
$$

By Lemma 5.1(1), the composite

$$
P^{8 n-2}(2) \stackrel{g}{\longrightarrow} P^{4 n}(2) \longrightarrow S^{4 n}
$$


is nullhomotopic and so $g$ lifts to $F^{4 n}\{2\}$. Let $\tilde{g}: P^{8 n-2}(2) \rightarrow F^{4 n}\{2\}$ be a lifting of $g$ and let $\bar{g}$ denote the composite

$$
P^{8 n-2}(2) \stackrel{\tilde{g}}{\longrightarrow} F^{4 n}\{2\} \longrightarrow Q\left(S^{4 n-1}\right) .
$$

From the homotopy commutative diagram (5-1), $\bar{g}$ lifts to $\Omega Q\left(S^{4 n}\right)$. By Lemma 5.1(2), $\bar{g}$ lifts further to $\Omega S^{4 n}$. It follows that there is a map $f: P^{8 n-2}(2) \rightarrow E$ such that the composite

$$
P^{8 n-2}(2) \stackrel{f}{\longrightarrow} E \longrightarrow F^{4 n}\{2\} \longrightarrow P^{4 n}(2)
$$

is homotopic to $g$. Thus $\operatorname{Ker}\left(\left[P^{8 n-2}(2), P^{4 n}(2)\right] \rightarrow\left\{P^{8 n-2}(2), P^{4 n}(2)\right\}\right)$ is contained in

$$
\operatorname{Im}\left(\mathbb{Z} / 2 \oplus \mathbb{Z} / 2 \rightarrow\left[P^{8 n-2}(2), F^{4 n}\{2\}\right] \rightarrow\left[P^{8 n-2}(2), P^{4 n}(2)\right]\right) .
$$

Here, by Lemma $5.2, \mathbb{Z} / 2 \oplus \mathbb{Z} / 2$ refers to the direct sum of a copy of $\mathbb{Z} / 2$ given in the exact sequence (5-3) and a copy of $\mathbb{Z} / 2$ generated by the homotopy class of the composite

$$
P^{8 n-2}(2) \stackrel{\text { pinch }}{\longrightarrow} S^{8 n-2} \stackrel{\lambda_{4 n}}{\longrightarrow} F^{4 n}\{2\},
$$

where $\lambda_{4 n}: S^{8 n-2} \rightarrow F^{4 n}\{2\}$ induces an isomorphism on $H_{8 n-2}$.

Let $\bar{\lambda}_{4 n}: P^{8 n-2}(2) \rightarrow P^{4 n}(2)$ be the composite

$$
P^{8 n-2}(2) \stackrel{\text { pinch }}{\longrightarrow} S^{8 n-2} \stackrel{\lambda_{4 n}}{\longrightarrow} F^{4 n}\{2\} \longrightarrow P^{4 n}(2) .
$$

Since $\left.\bar{\lambda}_{4 n}\right|_{S^{8 n-3}}$ is trivial but $\left.\delta_{4 n}\right|_{8 n-3}$ is essential (because $\omega_{4 n-1}$ is not divisible by 2 ), we have $\left[\bar{\lambda}_{4 n}\right] \neq\left[\delta_{4 n}\right]$. Thus the elements $\left\{\left[\bar{\lambda}_{4 n}\right],\left[\delta_{4 n}\right]\right\}$ generate a subgroup ${ }^{2}$ of $\left[P^{8 n-2}(2), P^{4 n}(2)\right]$ containing

$$
\operatorname{Ker}\left(\left[P^{8 n-2}(2), P^{4 n}(2)\right] \rightarrow\left\{P^{8 n-2}(2), P^{4 n}(2)\right\}\right) .
$$

Since

$$
\left[S_{2} \mid\right] \in \operatorname{Ker}\left(\left[P^{8 n-2}(2), P^{4 n}(2)\right] \rightarrow\left\{P^{8 n-2}(2), P^{4 n}(2)\right\}\right),
$$

we have $\left[S_{2} \mid\right]=\left[\bar{\lambda}_{4 n}\right],\left[\delta_{4 n}\right]$ or $\left[\bar{\lambda}_{4 n}+\delta_{4 n}\right]$.

By Lemma 4.3,

$$
\delta_{4 n} \circ(\eta \wedge \mathrm{id}): P^{8 n-1}(2) \rightarrow P^{4 n}(2)
$$

is nullhomotopic.

${ }^{2}$ We do not claim that $\left[\bar{\lambda}_{4 n}\right]$ lies in $\operatorname{Ker}\left(\left[P^{8 n-2}(2), P^{4 n}(2)\right] \rightarrow\left\{P^{8 n-2}(2), P^{4 n}(2)\right\}\right)$. What we claim is that $\operatorname{Ker}\left(\left[P^{8 n-2}(2), P^{4 n}(2)\right] \rightarrow\left\{P^{8 n-2}(2), P^{4 n}(2)\right\}\right)$ is controlled by linear combinations of $\left[\bar{\lambda}_{4 n}\right]$ and $\left[\delta_{4 n}\right]$. 
Along the lines in the proof of Theorem 3.6, and using the properties that $\bar{\lambda}_{4 n}$ factors through $S^{8 n-2}$ and any map $S^{8 n-3} \rightarrow \Omega P^{4 n}(2)$ having nontrivial Hurewicz image is of order 4 [10], the composite (5-2) is nullhomotopic if $S_{2} \mid$ is replaced by $\bar{\lambda}_{4 n}: P^{8 n-3}(2) \rightarrow \Omega P^{4 n}(2)$. The proof is finished.

Acknowledgements The main result (Theorem 1.1) is supported by Russian Scientific Foundation, grant N 14-21-00035. The last author is also partially supported by the Singapore Ministry of Education research grant (AcRF Tier 1 WBS No. R-146-000190-112) and a grant (No. 11329101) of NSFC of China.

\section{References}

[1] M G Barratt, Homotopy ringoids and homotopy groups, Quart. J. Math., Oxford Ser. 5 (1954) 271-290 MR0073178

[2] M G Barratt, Spaces of finite characteristic, Quart. J. Math. Oxford Ser. 11 (1960) 124-136 MR0120647

[3] M G Barratt, ME Mahowald, The metastable homotopy of $\mathrm{O}(n)$, Bull. Amer. Math. Soc. 70 (1964) 758-760 MR0182004

[4] HJ Baues, Quadratic functors and metastable homotopy, J. Pure Appl. Algebra 91 (1994) 49-107 MR1255923

[5] C-F Bödigheimer, Stable splittings of mapping spaces, from: "Algebraic topology", (H R Miller, D C Ravenel, editors), Lecture Notes in Math. 1286, Springer, Berlin (1987) 174-187 MR922926

[6] F R Cohen, The unstable decomposition of $\Omega^{2} \Sigma^{2} X$ and its applications, Math. Z. 182 (1983) 553-568 MR701370

[7] F R Cohen, A course in some aspects of classical homotopy theory, from: "Algebraic topology", (H R Miller, D C Ravenel, editors), Lecture Notes in Math. 1286, Springer, Berlin (1987) 1-92 MR922923

[8] F R Cohen, On combinatorial group theory in homotopy, from: "Homotopy theory and its applications", (A Adem, R J Milgram, D C Ravenel, editors), Contemp. Math. 188, Amer. Math. Soc. (1995) 57-63 MR1349129

[9] F R Cohen, R Mikhailov, J Wu, A combinatorial approach to the exponents of Moore spaces, preprint (2015) arXiv:1506.00948

[10] F R Cohen, J Wu, A remark on the homotopy groups of $\Sigma^{n} \mathbf{R} \mathrm{P}^{2}$, from: "The Čech centennial”, (M Cenkl, H Miller, editors), Contemp. Math. 181, Amer. Math. Soc. (1995) 65-81 MR1320988

[11] E Dyer, R K Lashof, Homology of iterated loop spaces, Amer. J. Math. 84 (1962) 35-88 MR0141112 
[12] PS Green, RA Holzsager, Secondary operations in $K$-theory and applications to metastable homotopy, Illinois J. Math. 16 (1972) 415-422 MR0326725

[13] I M James, On the iterated suspension, Quart. J. Math., Oxford Ser. 5 (1954) 1-10 MR0061836

[14] M Mahowald, Some Whitehead products in $S^{n}$, Topology 4 (1965) 17-26 MR0178467

[15] M Mahowald, The metastable homotopy of $S^{n}$, Mem. Amer. Math. Soc. 72, Providence, R.I. (1967) MR0236923

[16] M Mahowald, On the metastable homotopy of $\mathrm{O}(n)$, Proc. Amer. Math. Soc. 19 (1968) 639-641 MR0225324

[17] K Morisugi, Metastable homotopy groups of $\operatorname{Sp}(n)$, J. Math. Kyoto Univ. 27 (1987) 367-380 MR898535

[18] K Morisugi, J Mukai, Lifting to mod 2 Moore spaces, J. Math. Soc. Japan 52 (2000) 515-533 MR1760602

[19] D A Tipple, A note on the metastable homotopy groups of torsion spheres, Bull. London Math. Soc. 3 (1971) 303-306 MR0298661

[20] J Wu, On combinatorial calculations for the James-Hopf maps, Topology 37 (1998) 1011-1023 MR1650426

[21] J Wu, Homotopy theory of the suspensions of the projective plane, Mem. Amer. Math. Soc. 769, Providence, RI (2003) MR1955357

[22] J Wu, On maps from loop suspensions to loop spaces and the shuffle relations on the Cohen groups, Mem. Amer. Math. Soc. 851, Providence, RI (2006) MR2203532

RM: Chebyshev Laboratory, St. Petersburg State University

14th Line, 29b, St. Petersburg, 199178, Russia

RM: St. Petersburg Department of Steklov Mathematical Institute

JW: Department of Mathematics, National University of Singapore

10 Lower Kent Ridge Road, Singapore 119076

rmikhailov@mail.ru, matwuj@nus.edu.sg

http://www.math.nus.edu.sg/ matwujie

Received: 2 June 2015 Revised: 20 September 2015 
